\title{
BACKGROUND MEASUREMENTS DURING PEP-II COMMISSIONING ${ }^{*}$
}

$\underline{\text { T. Mattison }}^{\#}$, D. Aston, B. Byers, D. Coupal, H. Destaebler, T. Fieguth, L. Keller, W. Kozanecki, W.R. Nelson, M. Petree, S. Petrak, S. Shapiro, A. Snyder, M. Sullivan, S. Wagner, SLAC, A. Boucham, D. Boutigny, Y. Karyotakis, J-Y. Nief, P. Petitpas, V. Tisserand, K. Zachariadou, Annecy, C. Goodenough, T. Lanting, British Columbia, A. Hasan, A. McKemey, Brunel,

S. Devmal, T. Geld, B. Meadows, M. Sokoloff, Cincinnati, T. Borak, R. Malchow, W. Toki, Colorado State, K. Benabed, N. Treps, Ecole Polytechique, F. Goozen, J. Kadyk, R. Kerth, N. Roe, M. Ronan, LBNL, R. Cizeron, R. Cousin, A. Durand, G. Fubiani, V. LePeltier, S. Sen, S. Trincaz-Duvoid, A. Valassi, G. Wormser, Orsay, F. LeDiberder, S. Versillé, Paris VI\&VII, R. Aleksan, G. DeDomenico, S. Emery, J-C. Faivre, A. Gaidot, B. Mayer, Saclay, J. Beach, P. Burchat, Ch. Cheng, X. Huynh, D. Kirkby, T. Meyer, E. Nehrlich, C. Roat, A. Soha, H. Tanaka, Stanford, S. Berridge, W. Bugg, J. Hargis, A. Weidemann, Tennessee, C. Hast, E. Potter, V. Sharma, UCSD, H. Band, J. Johnson, Wisconsin

\section{Abstract}

A variety of background detectors were installed at the interaction point of PEP-II for measurements of machine backgrounds during commissioning. Results from these detectors, machine experiments, and simulations have been used to reduce the backgrounds at PEP-II before the installation of the BaBar physics detector.

\section{INTRODUCTION}

The PEP-II collider has a design luminosity of $3 \times 10^{33}$ $\mathrm{cm}^{-2} \mathrm{~s}^{-1}$, which requires beam currents of 1 and 2 amperes in the rings. The synchrotron radiation flux from the magnetic beam-separation scheme, and the particle loss rate from beam-gas scattering, are potential sources of high backgrounds for the BaBar detector. Many detectors were installed at the interaction point (IP) to measure the backgrounds, from the start of PEP-II commissioning. The background simulations used in the design of PEP-II were enhanced to help understand the measurements. Dedicated beam experiments were done to diagnose and reduce the backgrounds.

\section{DETECTORS}

An X-ray spectrometer measured the synchrotron radiation (SR) spectrum. A $3 \times 3 \times 2 \mathrm{~mm}$ cadmium-zinc-telluride crystal was enclosed in a copper collimator with $3^{\circ}$ acceptance and remotely movable lead and steel filters. The collimator was mounted on a remotely steerable TV camera. The readout was through a local pre-amplifier, remote post-amplifier, PC-based pulse-height analyzer, and rateto-voltage converter. Energy resolution was $4 \%$ at $6 \mathrm{keV}$ to $1 \%$ at $100 \mathrm{keV}$, where the efficiency began to fall off. The early commissioning IP beam pipe had poor SR masking, and rates $>1 \mathrm{kHz} / \mathrm{mA}$ were observed. Interest-

*Work supported iby U.S. Department of Energy contrace DEAC03-76SF00098, CEA, IN2P3, and PPARC. ingly, the spectra had prominent Bragg-scattering features, rather than the expected material fluorescence peaks. The final beam pipe had much better masking, with X-ray rates difficult to distinguish from lost-particle (LP) backgrounds.

PIN diodes measured both SR and LP backgrounds on the IP beam pipe. Pairs of $1 \mathrm{~cm}^{2}, 300 \mu$ thick reversebiased PIN diodes separated by $1 \mathrm{~mm}$ lead absorbers with a thermally coupled thermistor were mounted at 3 positions in $\mathrm{Z}$ and 8 in phi. Readout was 20-bit charge integrating ADCs with analog and digital filtering giving $15 \mathrm{pA}$ resolution on currents up to $1 \mu \mathrm{A}$ at $7.5 \mathrm{~Hz}$. An onboard microcontroller asynchronously passed data over a Controller Area Network bus to a VME single-board computer running vxWorks. The data was logged to disk by a Java server running on a SUN workstation. Real-time data for beam-tuning and background experiments was also available. In addition, the increase in leakage current over time measured the integrated radiation dose to the diodes, calibrated by the offline integrated dose.

A BaBar double-sided silicon strip detector with final radiation-hard electronics and full readout chain measured occupancies next to the beam pipe. Occupancies were initially very high but decreased to $0.5-1 \%$ per $100 \mathrm{~mA}$ of current for both beams by the end of commissioning. Large steps in the detector leakage current were observed over the running period. These were due to bursts of radiation causing bias voltage to punch through strip capacitors, inducing p-stop shorts. Bias voltage to the BaBar silicon vertex tracker will be turned off during injection to avoid this problem.

The Mini-TPC detector gave fine-grain 3D tracking information from 4.5 to $10 \mathrm{~cm}$ in radius (less a $110^{\circ}$ wedge for the silicon strip) and $10 \mathrm{~cm}$ in Z. It had 6 sectors of 8 radius rows of 4 phi cathode pads (trapezoidal to maximize charge sharing), and operated with a drift field of 200 $\mathrm{V} / \mathrm{cm}$ at a gas gain of 50000 . The gate grid normally had $\pm 220 \mathrm{~V}$ on alternate wires to reduce space-charge effects 
and was opened for $20 \mu \mathrm{s}$ at a few $\mathrm{Hz}$ for readout. The pad pre-amps were $5 \mathrm{~m}$ away, and $30 \mathrm{MHz}$ FADCs digitized the data, which was recorded to disk on a microVax. Resolution from a test-beam at CERN was $25 \%$ for $\mathrm{dE} / \mathrm{dx}$, $70 \mu \times \sqrt{L_{c m}} \oplus 170 \mu$ in Z, $210 \mu$ in R-phi, and 9 and 4 mrad in polar and azimuthal angles. An event display and the hit and track counts were available in real time, and the chamber current was also available as a hardware signal. A detailed Monte Carlo simulation included a complete digitization in the miniTPC.

The Straw Chamber (originally built for the Crystal Ball) was 544 aluminum tubes, $4 \mathrm{~mm}$ in diameter, $34 \mathrm{~cm}$ long, in two double layers at 13 and $17 \mathrm{~cm}$ radius from the beam. The signal from both ends of each wire was amplified then recorded by analog storage, multiplexing, and a NIM ADC combined with a scanner-memory module in a CAMAC crate controlled by a PC. The chamber current also gave a real-time measurement of the radiation level.

The Crystal Ring measured the MeV photon background using $12 \mathrm{CsI}(\mathrm{Na})$ crystals with photomultiplier tube (PMT) readout. The crystals were $6 \times 6 \times 15 \mathrm{~cm}$, at radius $30 \mathrm{~cm}$ from the beam, on a trolley that scanned \pm 80 $\mathrm{cm}$ in Z. A RAID 8235 processor running EP/LX controlled a CAMAC crate with FERA ADCs that could be self-triggered for calibration with the $1.46 \mathrm{MeV}$ photons from $\mathrm{KCl}$ salt-substitute. This system also measured background photon spectra at low beam current. The PMT current was used as the background measurement at higher beam current, since the spectrum was dominated by pileup and the PMT voltage was limited by anode heating. A fiber-optic and LED pulser system propagated the calibration from the source+ADC system to the PMT current system at lower voltage.

The mini-Stand-Off-Box (mSOB) background detector simulated the 6000 liter water tank with 10000 PMTs on one surface that is the external part of the BaBar Cherenkov particle identification system. The mSOB water tank was $1 \mathrm{ft}^{3}$ with a quartz window at one face, allowing 48 PMTs to view the Cherenkov light created in the water. A CAMAC ADC system with 100-500 ns gates was controlled by a small Unix workstation via GPIB. The backgrounds were primarily $\mathrm{MeV}$ photons which Compton scatter, making electrons just above Cherenkov threshold, typically producing a single photoelectron in a single tube. Since the rate was high, in later runs a single $\mathrm{mSOB}$ tube was fed into a frequency to voltage converter for real-time measurements. This system was also used with $(3 \text { in })^{3}$ lead-glass blocks glued to PMTs, with a sensitivity similar to the $\mathrm{mSOB}$ but easier to manipulate. The mSOB single-tube rate extrapolated to $1 \mathrm{MHz}$ for the HER and $10 \mathrm{MHz}$ for the LER at design currents, motivating the construction of shielding upstream of BaBar.

The BaBar Calorimeter Prototype detector measured the photon background from $20 \mathrm{MeV}$ to a few $\mathrm{GeV}$. It was four CsI crystals mounted near the future BaBar endcap calorimeter location, read out by PMTs via scalars and
ADCs in a CAMAC crate controlled by a Macintosh. The single crystal rate was $140 \mathrm{~Hz}$ with a $100 \mathrm{MeV}$ threshold for a LER current of $300 \mathrm{~mA}$ in February 1999. A drop in the light yield of about $30 \%$ has been found after an exposure estimated to be a few hundred rads.

The instrumented flux return (IFR) background detector simulated the outer layers of the BaBar IFR. Eight resistive plate chambers (RPCs) about $(1 \mathrm{~m})^{2}$ and operated at 8 $\mathrm{kV}$ alternated with 1.25 in steel plates, with the first and last layers covered with 0.25 inch of steel. Counting rates as a function of position were recorded using BaBar IFR electronics and a CAMAC crate controlled by a PC. Early data motivated the addition of shielding on the two exposed edges of the chambers, and the corresponding areas of BaBar, and shielding in the PEP-II tunnels.

An array of 8 RadFETs was positioned at the future inner radius of the $\mathrm{BaBar} \mathrm{CsI}$ calorimeter to measure the integrated dose via threshold shifts. From November 1998 to February 1999, a dose of about 600 rads was accumulated in unshielded devices.

\section{SIMULATIONS}

The program SYNC_BKG traced the beam envelope through the magnets near the IP, calculated the number and spectrum of SR photons from each magnet, and projected them to apertures that modelled the beam pipe. This was input to MASKING, which used EGS4 to calculate the number, energy, and angle distribution of the photons that scatter from, for instance, a mask tip and penetrate the IP beam pipe to register as background in the SVT. MASKING could also generate an output file of photons to be used as input to detector simulations.

Lost-particle rates from bremsstrahlung and Coulomb scattering were calculated with the LP_TURTLE Monte Carlo, derived from Decay TURTLE. The optics and apertures of both rings were modeled for their entire circumferences. Information about the trajectory of particles striking apertures near the IP was stored to file in the form of ntuples. LP_TURTLE output was input for collimator placement studies, multi-turn and longitudinal beamdynamics simulations, and detector simulations.

The BaBar detector simulation with GEANT3 (BBSIM) includes the PEP-II beam pipe, magnet material, and magnetic fields to $8 \mathrm{~m}$ from the IP. An equally complete simulation was done of the IP configuration used for early commissioning of the HER, and of the various background detectors. Rays which hit TURTLE apertures close to the IP were backed out to $8 \mathrm{~m}$ and tracked again in BBSIM since it has a more detailed 3D model of the apertures in the detector. BBSIM modelled electromagnetic showering and energy deposition in detectors.

\section{EXPERIMENTS}

Initially backgrounds were much higher than expected from simulations. Turning off distant vacuum pumps increased the background, indicating that distant beam-gas 
scattering was important, which had not been included in the initial LP_TURTLE simulation. Reducing the RF voltage to the point where the quantum lifetime was short did not increase the backgrounds, indicating that distant bremsstrahlung was not dominant.

Backgrounds were sensitive to beam steering at the IP, particularly changes in IP angle, which corresponds to beam position in the high-beta quads. In the initial IP geometry with poor masking, extreme steering bumps varied the SR contribution differently from the LP contribution, which allowed them to be separated in some detectors. Backgrounds also decreased in some detectors when the IP beta function was increased, decreasing the beam size in the quads. Later in commissioning, single-sided fixed collimators were installed in the HER $350 \mathrm{~m}$ upstream of the IP, at points where the beam could be bumped close the aperture. This reduced backgrounds, and the sensitivity to steering and beta function.

Measuring the backgrounds as a function of beam current was very informative. Synchrotron radiation and beam-gas scattering on base pressure and give linear current dependence, and beam-gas scattering on dynamic pressure induced by the beam gives a quadratic contribution. The quadratic term was dominant at high current.

There were many non-evaporable getter (NEG) pumps in the IR, which expelled hydrogen when they were heated for regeneration. Heating a NEG pump produced a large localized pressure bump of known composition, with magnitude measured by the nearby ion pumps or by the change in beam lifetime. The increase in backgrounds from the pressure bump allowed a precision check of the simulation. The agreement was good.

\section{CONCLUSIONS}

Measured backgrounds from the January 1998 configuration of the HER were consistent with a factor of 7-10 more background at design current than estimated in the BaBar design report [1]. The measurements were consistent (within a factor of 1.5-2) with a simulation using measured pressure profiles rather than a uniform 1 nTorr, LP_TURTLE simulation of the full ring rather than just the IP and a short arc region, and BBSIM shower simulation including all the IP quads rather than just those inside the detector. See figure 1. Collimators reduced the HER arc contribution by a factor of 2-5 in the final HER configuration.

Measured backgrounds from the LER (without collimators) were comparable to the HER at the same current. The absolute LER background levels agreed with the extended simulation to within factors of 2-5; work is in progress to clarify the origin of these differences.

Extrapolating present measurements to design currents, LER background would dominate. A substantial reduction in LER background is expected from distant Coulomb collimators presently being installed. Some reduction is expected from the larger aperture of the final Q2 vacuum chamber. The LER vacuum will improve with further scrubbing, and a program of vacuum improvements in the LER is underway.

\section{REFERENCES}

[1] BaBar Technical Design Report, SLAC-R-95-457, March 1995

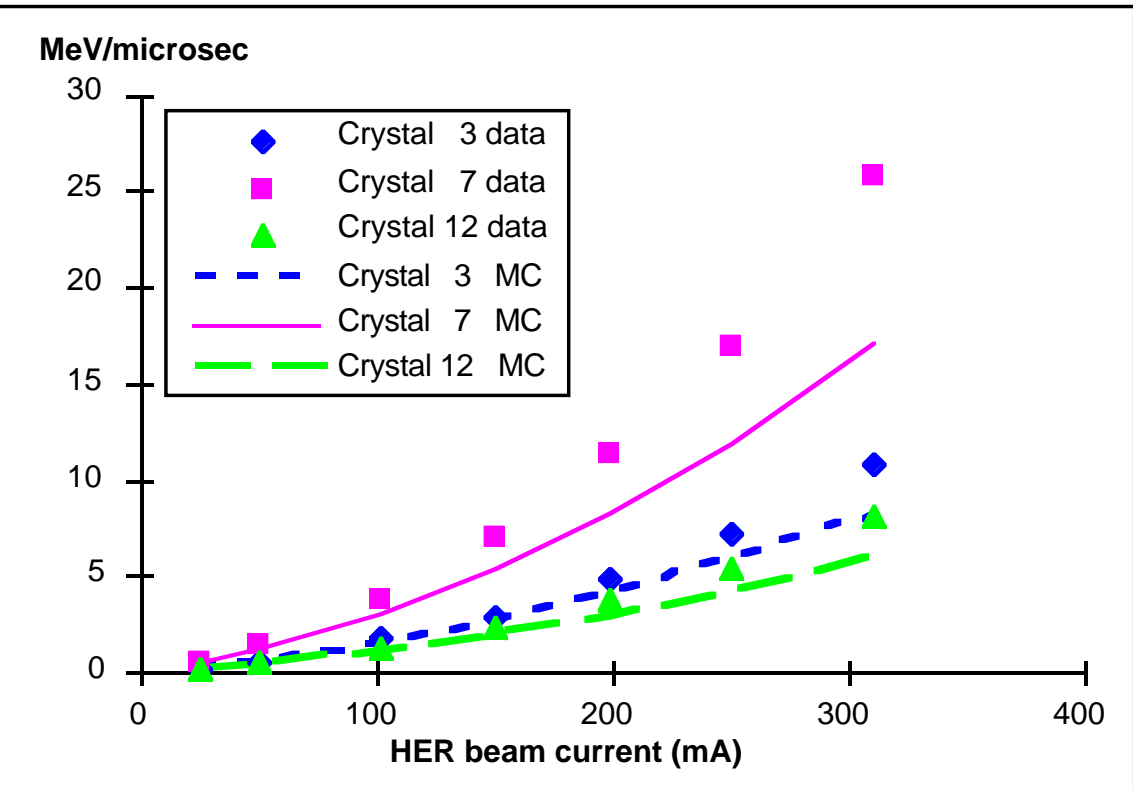

Figure 1: Comparison of measured and simulated backgrounds in the Crystal Ring detector as a function of beam current during HER commissioning in January 1998. 